\title{
The Effect of Viscosity on Magnetic Generation in Solar Plasmas
}

\author{
Zhiliang Yang $^{1}$ and Hairong Jing $^{1}$ \\ ${ }^{1}$ Department of Astronomy, Beijing Normal University, Beijing 100875, P. R. China \\ email: zlyang@bnu.edu.cn
}

\begin{abstract}
Based on the dynamics of charged particles in plasma with the two-fluid description, the equation for magnetic field generation and maintenance is derived. The nature of magnetic field in cosmic plasma and the generation can be understood from the equation. The ions and electrons are considered as two independent fluids with the collision connected. Due to the different viscosity between ions and electrons, there will be a velocity difference in ions and electrons. This causes a current in the plasma and generates magnetic field. IN the plasma system, the differential velocities and the viscous forces of the electrons are the source of magnetic field.
\end{abstract}

Keywords. Sun: magnetic fields, Plasmas

\section{The nature of magnetic field generated in plasma}

In the universe magnetic fields are detected everywhere (see some of the reviews (Han, et al. 2002; Schekochihin, et al. 2003). The magnetic fields play a curious role in astrophysics for its commonplace and poorly understood. Dynamo theory is the theory that describe the existence of magnetic fields in conducting fluid masses. Dynamo problems are of two kinds, the kinematic dynamos as the fluid velocities are regarded as given and the hydromagnetic dynamos with the fluid velocities are determined by equations of motion including forces of magnetic origin. The reviews on dynamo theories could be seen in a lot of literatures (Cowling, 1981; Schmitt, 1993).

Plasma is the collection of charged particles with a neutralized charge as a whole. Considering plasma as two-fluid of ions and electrons (Huba \& Fedder, 1993), We can get the magnetic field at $\mathrm{r}$ in laboratory system

$$
\mathbf{B}=\Sigma \frac{m u_{0}\left(n_{i} e \mathbf{v}_{\mathrm{i}}-n_{e} e \mathbf{v}_{\mathrm{e}}\right) \times \mathbf{r}}{4 \pi r^{3}}
$$

$n_{i} e \mathbf{v}_{\mathbf{i}}-n_{e} e \mathbf{v}_{\mathbf{e}}$ is the current in the plasma. We can see that the condition for the generation of magnetic field is $n_{i} \mathbf{v}_{\mathbf{i}}-n_{c} \mathbf{v}_{\mathbf{e}} \neq 0$. we can get:

$$
\mathbf{B}=\Sigma \frac{m u_{0} n e\left(\mathbf{v}_{\mathbf{i}}-\mathbf{v}_{\mathbf{e}}\right) \times \mathbf{r}}{4 \pi r^{3}}
$$

\section{Assumptions and Equations}

Suppose the plasma consists of protons and electrons only. For the case that higher ions existed in plasma, we can easily get the result following the way we discussed. The plasma fluid equations are given by:

$$
\frac{d \mathbf{v}_{\alpha}}{d t}=\frac{q_{\alpha}}{m_{\alpha}}\left(\mathbf{E}+\frac{1}{c} \mathbf{v}_{\alpha} \times \mathbf{B}\right)-v_{\alpha \beta}\left(\mathbf{v}_{\alpha}-\mathbf{v}_{\beta}\right)-\frac{\nabla P_{\alpha}}{n_{\alpha} m_{\alpha}}+F_{\alpha}
$$


where $\mathbf{v}_{\alpha}, \mathbf{v}_{\beta}$ refers to electrons and/or protons $(e, i), \mathbf{F}_{\alpha}$ is the external force acted on protons or electrons except the electromagnetic force.

The electromagnetic induction equation:

$\frac{\partial \mathbf{B}}{\partial t}=\nabla \times\left(\mathbf{v}_{i} \times \mathbf{B}\right)-\frac{1}{n e} \nabla \times(\mathbf{J} \times \mathbf{B})+\eta \nabla^{2} \mathbf{B}+\frac{c \nabla \times \nabla \mathbf{P}_{e}}{n e}-\frac{c}{e} \nabla \times \mathbf{F}_{e}-\frac{c}{e} \nabla \times\left[\left(\mathbf{v}_{e} \cdot \nabla\right) \mathbf{v}_{e}\right]$

The first term of equation (2.2) $\nabla \times\left(\mathbf{v}_{i} \times \mathbf{B}\right)$ is the convective term, the second term $\frac{1}{n e} \nabla \times(\mathbf{J} \times \mathbf{B})$ is the Hall term, the third term $-\frac{c m_{i} \nu_{i e}}{n e^{2}} \nabla \times \mathbf{J}$ is magnetic diffusion (the resistivity is $\eta=\nu_{i c} c^{2} / \omega_{i e}^{2}$, where $\omega_{i e}^{2}=4 \pi n e^{2} / m_{e}$, and the terms $-\frac{c}{e} \nabla \times \mathbf{F}_{e}$ and $\frac{c \nabla \times \nabla \mathbf{P}_{e}}{n e}$ is the source term for the magnetic field. In regular flow, $-\frac{c}{e} \nabla \times\left[\left(\mathbf{v}_{e} \cdot \nabla\right) \mathbf{v}_{e}\right.$ is ignored in the assumption of incompressible fluid.

\section{The Viscosity on the Dynamo Effect in Differential Rotated Plasma}

Eventually, the famous Biermanns 'battery is one of the results of two-fluid model of a fully ionized plasma. If there is no magnetic field in the plasma, then $\mathbf{E}+\frac{\nabla P_{e}}{n_{e} e}=0$. In general stars, $\nabla \times \nabla P_{e}=0$. The Biermanns Battery is not important. The magnetic field should be generated from the different velocities of ions and electrons.

In the two-fluid model of fully ionized plasma, the viscosity difference between ions and electrons is the possible mechanism to cause the different velocities of ions and electrons. Since the motion of plasma is assumed as conditions, the motion of ions is a fixed condition, only the viscous force of electrons should be included in equation (2.2). The viscous force of electron can be expressed as:

$$
\mathbf{f}_{v i s-e}=\nu \nabla^{2} \mathbf{v}_{i}
$$

The magnetic induction equation (2.2) can be written as the following simple form with the Hall term ignored:

$$
\frac{\partial \mathbf{B}}{\partial t}=\nabla \times\left(\mathbf{V}_{i} \times \mathbf{B}\right)+\eta \nabla^{2} \mathbf{B}+-\frac{c m_{e}}{e} \nabla \times\left(\nu \nabla^{2} \mathbf{v}_{i}\right)
$$

In the spherical coordinate, we can get the magnetic field in the $\mathbf{r}$ and $\theta$ direction with the assumption $\mathbf{v}=v_{\varphi} \varphi$.

$$
\mathbf{B}_{\mathbf{r} \theta}=\frac{c \nu}{\eta e} \nabla \times v_{i-\varphi}=\frac{c m_{e}}{e} P_{m} \nabla \times v_{i-\varphi}
$$

The parameter $P_{m}$ is the magnetic Prandtl number defined as the ratio of viscous to magnetic diffusivity, $P_{m}=\frac{\nu}{\eta}$.

we can see that the magnetic generation is from $\nabla \times \mathbf{v}$ and depends on the Prandtl number.

As a example, we can estimate the poloidal component of magnetic field inside the sun. From the differential rotation inside the sun (Dikpati \& Charbonneau, 1999), we can estimate $\nabla \times \mathbf{v} \sim$ inside the sun. Using the Spitzer values for the viscosity and magnetic diffusivity of a fully ionized plasma, one finds

$$
P_{m} \sim 2.6 \times 10^{-5} T^{4} / n
$$

Where $T$ is temperature. In the case of a partially ionized gas with neutral dominated viscosity, the formula for $P_{m}$ is

$$
P_{m} \sim 1.7 \times 10^{-7} T^{2} / n
$$


In solar plasma, the Prandtl number is $P_{m} \sim 10^{10}-10^{14}$. With small differential velocity $\nabla \times v$ we can get strong magnetic field.

\section{Summary and Discussions}

In this poster, we stress that the current in plasma is the result of different velocities of ions and electrons. The difference is due to the difference of viscosity for ions and electrons. In the two-fluid model, the ions and electrons are two fluids. Each has own viscosity. The kinematic viscosity $v$ is anti-proportional to the density of fluid. The viscosity for ions is smaller then that for electrons due to their different mass. In the frame of ions or plasma, the viscous force of electrons is the main mechanism for magnetic generation. The reverse process is the dissipation of current, which is the collision between ions and electrons and decreases the velocity difference between ions and electrons. This is the Ohms dissipation.

\section{Acknowledgements}

The research is supported by NFSC under grant number 10273003 and the 973 Project of China under grant number G200078400.

\section{References}

Han, J.-L. \& Wieleninsk, R. 2002, Chin. J. Astron. Astrophys. 2, 293

Schekochihin, A. A., Cowley, S. C., Taylor, S. F., Maron, J. L., \& McWilliams, J. C. 2003, Astrophys. J., Astro-ph/0312046

Cowling, T. G. 1981, Ann. Rev. Astron. Astrophys., 19, 115

Schmitt, D. 1993, The Cosmic Dynamo, IAU, Printed in the Netherlands, p1

Huba, J. D. \& Fedder, J. A. 1993, Phys. Fluids B., Vol. 5, No. 10, 3779

Dikpati, M. \& Charbonneau, P. 1999, Astrophys. J., 518, 508 